\title{
NEREIDIDAE (ANNELIDA, POLYCHAETA) DA COSTA NORDESTE DO BRASIL. II. GÊNEROS NAMALYCASTIS, CERATOCEPHALE, LAEONEREIS E RULLIERINEREIS
}

\author{
Cinthya S. G. Santos ${ }^{1,2}$ \\ Paulo C. Lana ${ }^{1}$
}

\begin{abstract}
NEREIDIDAE (ANNELIDA, POLYCHAETA) FROM THE NORTHEASTERN COAST OF THE BRAZIL. II. GENERA NAMALYCASTIS, CERATOCEPHALE, LAEONEREIS AND RULLIERINEREIS. Six polychaete species belonging to the genera Namalycastis Hartman, 1959, Ceratocephale Malmgren, 1867, Laeonereis Hartman, 1945, and Rullierinereis Pettibone, 1970 were recorded as part of a systematic survey of the family Nereididae in estuaries, exposed sandy beaches, shelly soft bottoms, atolls and coral reefs of the Brazilian northeastern coast. Two new species, Rullierinereis auxiliadorae, from Ceará coast and Ceratocephale rocaensis, from Atol das Rocas, are described.
\end{abstract}

KEYWORDS. Taxonomy, Nereididae, Northeastern Brazil.

\section{INTRODUÇÃO}

Até o presente, foram registradas 46 espécies de nereidídeos ao longo da costa brasileira. As primeiras referências remontam a Grube (1872), HANSEN (1882), KINBERG (1866) e TREADwell (1920, 1923, 1932), que descreveram várias espécies a partir de material proveniente das costas sudeste e sul. CORRÊA (1948) descreveu Lycastis siolii, proveniente do rio Tapajós, na Amazônia. Estudos mais abrangentes sobre os poliquetas do Brasil, alguns dos quais fazem menção aos nereidídeos, tiveram início na década de 70, (Nonato \& Luna, 1970; Orensanz \& Estivariz, 1971; Orensanz \& Gianuca, 1974; Rullier \& Amoureux, 1979; Lana, 1987; Lana \& Sovierzoski, 1987; Paiva, 1993; Morgado et al., 1994; SAntos et al., 1994; SANTOS \& LANA, 2000).

Das 10 espécies conhecidas do gênero Ceratocephale Malmgren, 1867, apenas C. oculata Banse, 1977 havia sido registrada para a costa brasileira por Lana (1987) e PaIVA (1993), respectivamente nas costas sul e sudeste. O gênero Namalycastis Hartman, 1959 foi registrado nas costa norte, sudeste e sul por KINBERG (1866), CORRÊA (1948), Hartman (1959), Lana (1987), Rebelo (1988) e Glasby (1999), totalizando 5 espécies inclusa N. macroplatis descrita por Glasby (1999) para a região norte. Glasby (1999), em sua revisão da subfamília Namanereidinae, reportou as seguintes espécies para a costa brasileira: N. abiuma (Grube, 1872), N. siolii (Corrêa, 1948), N. brevicornis (Audouin \& Edwards, 1833), N. senegalensis (Saint-Joseph, 1900) e N. macroplatis. O gênero Laeonereis Hartman, 1945 foi registrado para a costa do Rio de Janeiro por TREADWELl (1923); os demais registros, também concentrados nas costas sul e sudeste foram feitos por Orensanz \& Estivariz (1971), Orensanz \& Gianuca (1974) e Lana (1987). Adicionalmente SANTOS et al. (1994) registraram o gênero em estuários da costa

1. Centro de Estudos do Mar, Universidade Federal do Paraná, CP 002, 83255 000, Pontal do Paraná, PR

2. Pós-Graduação em Zoologia, Departamento de Zoologia, Universidade Federal do Paraná. (Bolsista CNPq, Doutorado). 
de Sergipe. O gênero Rullierinereis Pettibone, 1971, que abriga atualmente 10 espécies, é registrado pela primeira vez na costa brasileira.

Objetiva-se o levantamento taxonômico das espécies dos gêneros Namalycastis, Ceratocephale, Laeonereis e Rullierinereis no nordeste do Brasil.

\section{MATERIAL E MÉTODOS}

Os espécimes provenientes da plataforma continental brasileira foram obtidos a partir do Projeto Geocosta/ 1994, no Ceará e Projeto Algas/1980, na Paraíba. Essas amostras e aquelas provenientes de região sublitoral estuarina foram obtidas com um pegador de fundo do tipo "Van Veen" (HoLME \& McInTYRE, 1971). As coleções provenientes da região entre-marés, que inclui praias arenosas, areno-lodosas, rochosas, manguezais, costões e recifes, foram obtidas através de testemunhadores e por raspagem do material incrustado. Todo o material foi triado através de peneiras de $1,0 \mathrm{~mm}$ e $0,5 \mathrm{~mm}$, fixado em formol $4 \%$ e conservado em álcool $70 \%$. Algumas amostras foram anestesiadas com cloreto de magnésio a $8 \%$. Os procedimentos taxonômicos seguiram a rotina descrita por DAY (1967) e a nomenclatura utilizada para caracterização morfológica baseia-se em diversos autores (DAY, 1967; Hutchings \& TuRveY, 1982; Glasby, 1999). Para as descrições e ilustrações foram tomadas medidas da largura do prostômio e da largura do anel proboscidial oral dos exemplares em melhor estado. A altura dos parapódios foi estimada com base na distância entre o ponto de inserção do cirro dorsal e do cirro ventral (nos parapódios onde o cirro dorsal se desloca, a medida foi tomada na base da lígula notopodial). A largura do corpo, com e sem parapódios, foi medida no setígero 5. O material examinado encontra-se depositado na coleção de poliquetas do Centro de Estudos do Mar, Universidade Federal do Paraná (MCEM) e na coleção do Instituto de Biociências da Universidade Federal do Rio de Janeiro (UFJR).

\section{Namalycastis abiuma (Grube, 1872)}

(Figs. 1-6)

Lycastis abiuma GRUBE, 1872:47, 48.

Namalycastis abiuma; HaRTMAn, 1959:163-165, figs. 1- 4; REBELo, 1988:5-9, figs. 1 a-f; GlasBy, 1999:31, figs. 1c, 9,10 a-h.

Namalycastis aibiuma [sic]; Wu et al., 1985:45-46, fig.22, figs. a-i.

Comentários. A espécie foi originalmente descrita no gênero Lycastis, a partir de material proveniente de Florianópolis e subseqüentemente registrada em diversas partes do mundo, o que lhe conferiu o status de cosmopolita. GLASBY (1999) não conseguiu determinar limites morfológicos e geográficos que permitissem o desdobramento do táxon, que caracteriza-se pelos cirros dorsais posteriores triangulares, expandidos e duas vezes mais longos que o lóbulo neuropodial. Preferiu adotar uma solução de compromisso, abrigando os espécimes sob o nome informal de grupo Namalycastis abiuma. Este grupo incluiria espécimes provenientes de diversas partes do mundo, inclusive aqueles da costa brasileira. Ainda segundo Glasby (1999), apesar da impossibilidade de identificar diferentes espécies, as variações registradas nos diferentes espécimes observados são maiores do que aquelas normalmente atribuídas aos gêneros próximos de Namalycastis. Considerando a decisão de Glasby (1999) optamos por identificar o material proveniente do nordeste brasileiro como $N$. abiuma (figs. 1-6).

Ocorrência. Comumente encontrada na região entre-marés de bancos lodosos, associada a troncos caídos de árvores de manguezais. Na região estudada foi encontrada em raízes de Rhizophora mangle Linnaeus.

Distribuição. Ampla distribuição mundial, típica de áreas estuarinas tropicais e subtropicais. A espécie é exclusiva de águas quentes e de acordo com Glasby (1999), o grupo $N$. abiuma, incluindo as sinonímias propostas, possui distribuição circumglobal entre $30^{\circ} \mathrm{Ne} 30^{\circ} \mathrm{S}$. 

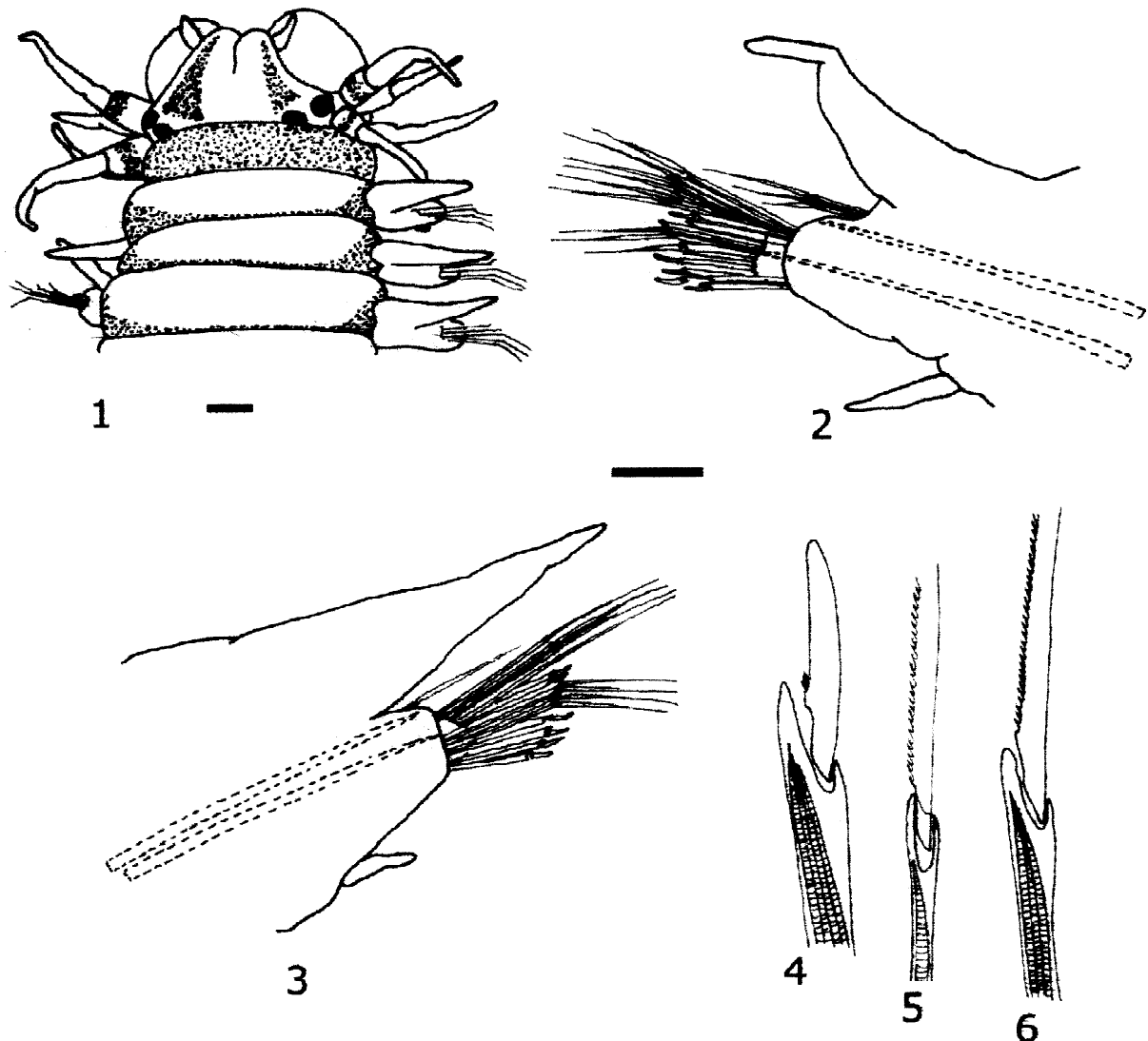

Figs. 1-6. Namalycastis abiuma (Grube, 1872), rio Piauí, Sergipe: 1, região anterior, vista dorsal; 2 , parapódio do setígero 37, vista anterior; 3, parapódio do setígero 78, vista posterior; 4, seta heterogonfa falcígera; 5, seta espinígera sesquigonfa; 6 , seta espinígera heterogonfa. Escalas: $500 \mu \mathrm{m}$, fig. 1; $100 \mu \mathrm{m}$, figs. 2,$3 ; 50 \mu \mathrm{m}$, figs. $4-6$.

Material examinado. BRASIL, Sergipe: Estuário do rio Piauí (MCEM 876, 1 ex.).

\section{Namalycastis macroplatis Glasby, 1999}

(Figs. 7-14)

Namalycastis macroplatis GLASBy, 1999:61, figs. 1c, 24, 25 a-g.

Comentários. Namalycastis macroplatis, com cirros dorsais posteriores delgados, 4 a 5 vezes mais longos que o lóbulo neuropodial e setas falcígeras restritas a alguns setígeros (figs. 7-14), difere de N. abiuma, que apresenta setas falcígeras heterogonfas supra- e subaciculares em todos os parapódios e cirros dorsais posteriores triangulares, robustos, duas vezes mais longos que o lóbulo neuropodial. $N$. brevicornis, registrada em 

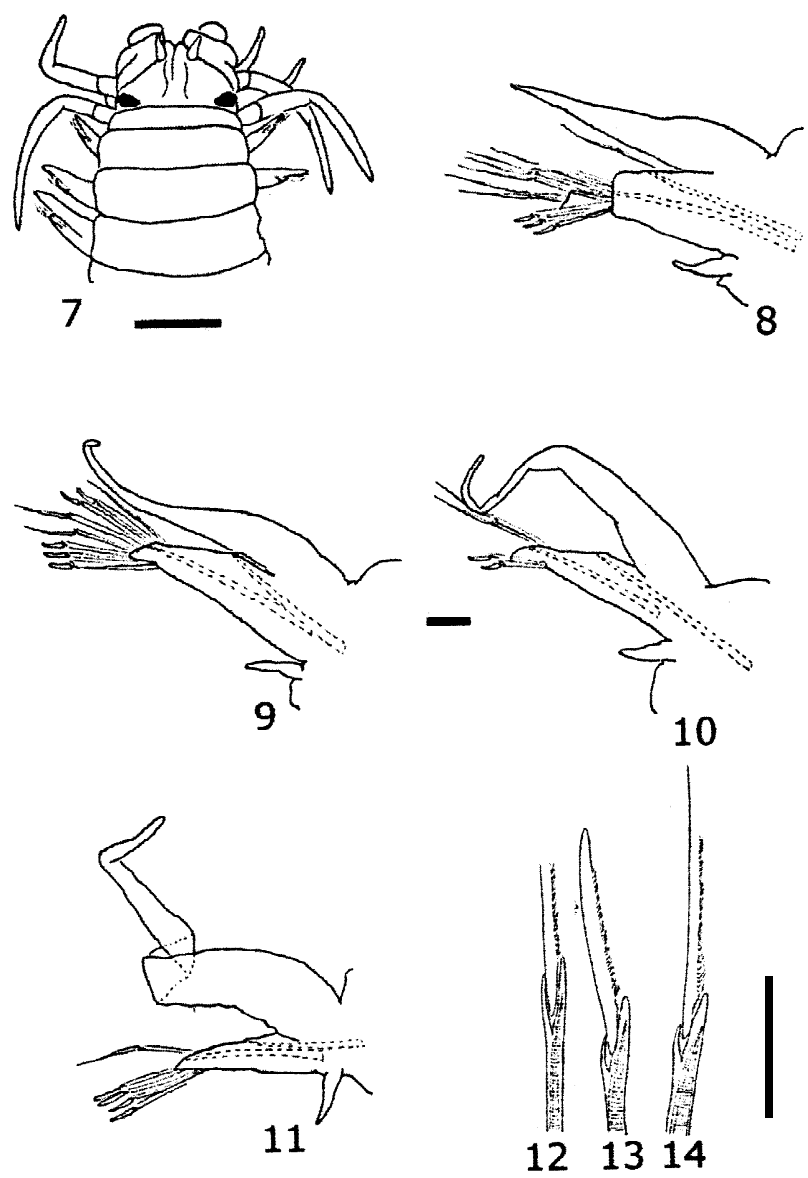

Figs. 7-14. Namalycastis macroplatis Glasby, 1999, rio Japaratuba, Sergipe: 7, região anterior, vista dorsal; 8, parapódio do setígero 9, vista anterior; 9, parapódio do setígero 30, vista anterior; 10, parapódio do setígero 66, vista anterior; 11 , parapódio do setígero 110 , vista anterior; 12 , seta espinígera sesquigonfa; 13 , seta falcígera heterogonfa; 14, seta espinígera heterogonfa. Escalas: $500 \mu \mathrm{m}$, fig.7; 100 $\mu \mathrm{m}$, figs. 8-11; $50 \mu \mathrm{m}$, figs. 12-14.

região entre-marés areno-lodosa na França e na Ilha de Marajó, difere de N. macroplatis por apresentar setas falcígeras heterogonfas supra- e subaciculares em todos os parapódios, setas falcígeras heterogonfas lisas e cirro dorsal aproximadamente do mesmo comprimento do lóbulo neuropodial em parapódios anteriores. N. siolii, registrada em rios da Região Amazônica, apresenta cirros dorsais posteriores triangulares, robustos e até duas vezes mais longos que o lóbulo neuropodial, ao contrário dos cirros dorsais delgados e muito mais longos que os lóbulos neuropodiais encontrados na região posterior de $N$. macroplatis. Em $N$. senegalensis os cirros dorsais posteriores apresentam um estreitamento abrupto na região subterminal, e em $N$. macroplatis o estreitamento é progressivo e menos pronunciado.

Wu et al. (1985) referiram N. longicirris (Takahashi, 1933) para a Região 
Amazônica. Segundo Glasby (1999), esta é restrita à localidade-tipo, ocorrendo em lagos, banhados e arrozais da China. Os cirros dorsais de $N$. longicirris são similares àqueles encontrados em $N$. macroplatis. No entanto, diferem pela presença de setas falcígeras heterogonfas nos feixes supra- e subaciculares em todos os parapódios de $N$. longicirris.

Variação. Os espécimens examinados (figs. 7-14) variam em relação ao material descrito por Glasby (1999) como N. macroplatis, por apresentarem uma seta falcígera subacicular em parapódios posteriores e pela ausência de pigmentação dorsal. Entretanto, esta variação não é considerada suficiente para eleição de uma nova espécie e o material é referido a $N$. macroplatis.

Ocorrência. Em substrato arenoso de região sublitoral, em estuário mixo- a oligohalino.

Distribuição. Além da localidade-tipo, a Ilha de Marajó, Belém, Pará, Brasil, ocorre no Suriname, Panamá e ora registrado na região Nordeste do Brasil (estuário do rio Japaratuba, em Sergipe e no Maranhão) ampliando a distribuição do táxon para o sul. Existe ainda um registro duvidoso para o Golfo de St. Lawrence, onde a espécie teria sido introduzida, fornecendo mais um exemplo de distribuição disjunta entre os Namanereidinae (GLASBY, 1999).

Material examinado. BRASIL, Maranhão: São Luís, Manguezal Parnauaçu-P8 (MCEM 877, 1 ex.); Sergipe: Estuário do rio Japaratuba (MCEM 878, 1 ex.; 878a 1 ex.; 879- 880, 2 exs cada).

\section{Ceratocephale oculata Banse, 1977}

(Figs. 15-18)

Ceratocephale loveni; DAY, 1973:38 [non MALMGREN, 1867].

Ceratocephale oculata BANSE, 1977:620, fig. 3; TAYLOR, 1984:9, figs. 6- 9; PAIVA, 1993:73.

Ceratocephale crosslandi; SANTOS et al., 1994:544.

Comentários. Ceratocephale oculata caracteriza-se pela presença de olhos, cirros ventrais duplos a partir do setígero 1 e setas exclusivamente espinígeras homogonfas (figs. 15-18). A espécie foi descrita a partir de material erroneamente referido a C. loveni Malmgren, 1867 por DAY (1973). SANTOS et al. (1994) registraramna em estuários de Sergipe, referindo-a equivocadamente a C. crosslandi (Monro, 1933). O material identificado por LANA (1987) na costa do Paraná como C. oculata foi reexaminado e identificado como Gymnonereis Horst, 1918, que difere de Ceratocephale pela presença de projeções laterais nos cirróforos.

Ocorrência. Em substrato síltico-argiloso com areia biodetrítica e salinidade entre 20 e 30, em região sublitoral estuarina.

Distribuição. Ilha Gorgona, Panamá; costa leste norte-americana (Carolina do Norte) até o Golfo do México; costa oeste do México; costa sudeste do Brasil. Na costa nordeste do Brasil foi registrada no sul de Sergipe, nos estuários dos rios Japaratuba, Piauí e Real e no recife de Timbabas na Bahia

Material examinado. BRASIL, Sergipe: estuário do rio Japaratuba ( MCEM 985, 6 exs; 1005, 1 ex.); estuário do rio Piauí (MCEM 963-984, 64 exs; 987, 1 ex.; 990-999, 35 exs); estuário do rio Real (MCEM 989-1004, 43 exs); Bahia: recife de Timbabas (UFRJ 95, 21 exs). 


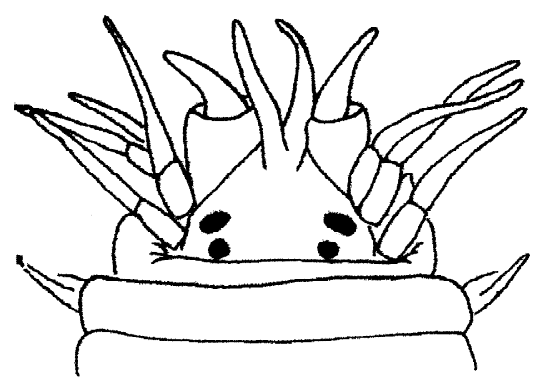

15
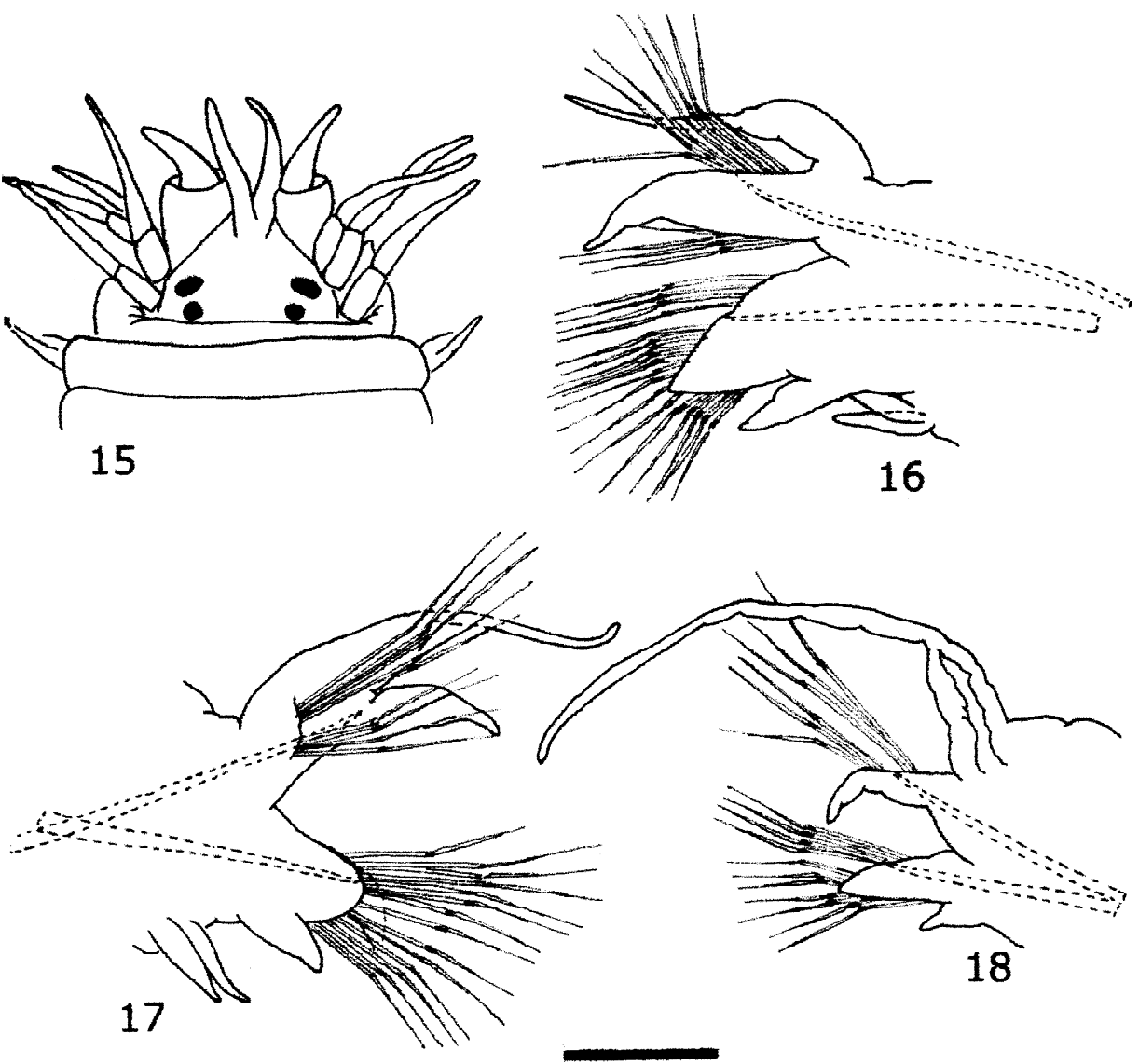

Figs. 15-18. Ceratocephale oculata Banse, 1977, rio Real, Sergipe: 15, Região anterior, vista dorsal; 16, parapódio do setígero 4, vista anterior; 17 , parapódio do setígero 14, vista posterior; 18 , parapódio do setígero 29, vista anterior. Escala: $200 \mu \mathrm{m}$, figs. 15-18.

\section{Ceratocephale rocaensis sp. nov.}

(Figs. 19-25)

Etimologia. $\mathrm{O}$ adjetivo específico faz referência à localidade-tipo, o Atol das Rocas, ao largo do Rio Grande do Norte.

Descrição. Entre os 6 espécimes examinados, apenas três estão completos apresentando entre 90 e 107 setígeros e medindo entre 12 e $17 \mathrm{~mm}$. O holótipo é um exemplar completo com 107 setígeros e $12 \mathrm{~mm}$ de comprimento, medindo $700 \mu \mathrm{m}$ de largura sem os parapódios e $1250 \mu \mathrm{m}$ com os parapódios, na altura do setígero 5. Coloração amarelo-esbranquiçada em álcool 70 \%. Prostômio anteriormente fendido; um par de antenas cirriformes atingindo metade do comprimento dos palpos; um par de palpos delicados, com palpóforos cilíndricos e longos e palpóstilos cônicos; dois pares de 

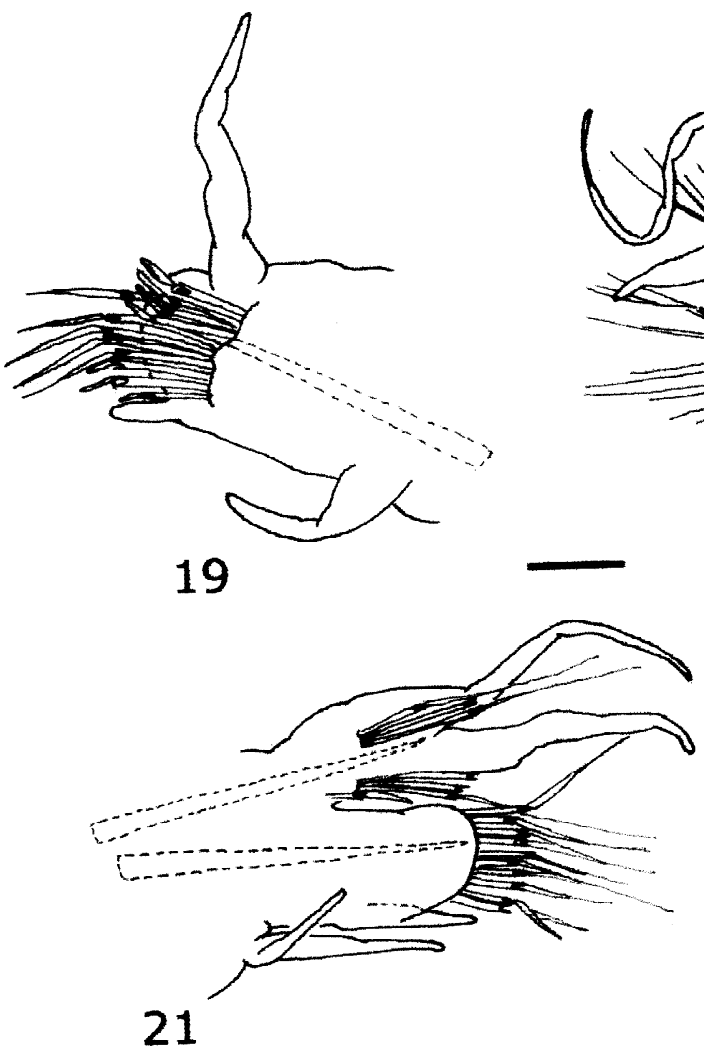

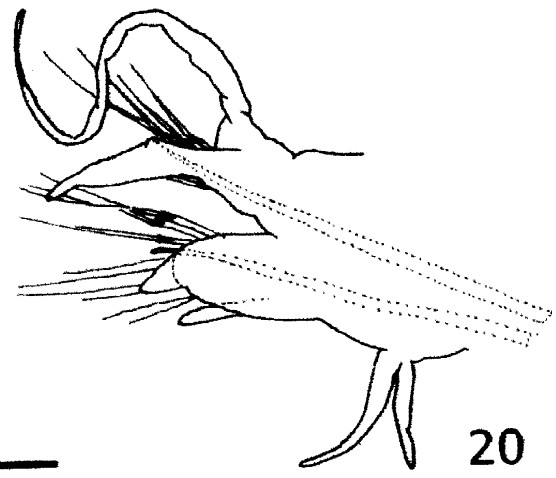

20

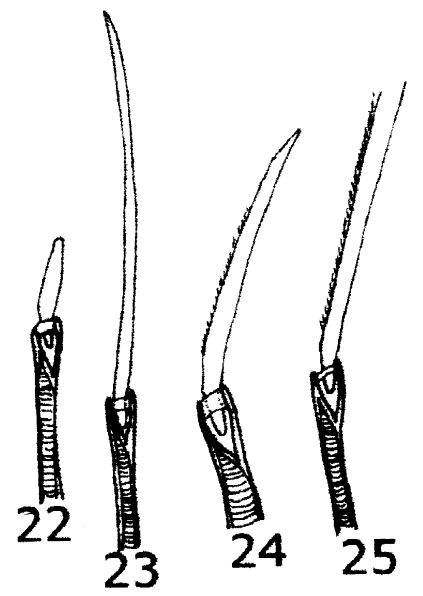

Figs. 19-25. Ceratocephale rocaensis sp. nov., holótipo: 19, parapódio 2, vista anterior; 20, parapódio 11, vista posterior; 21, parapódio 24, vista anterior; 22, seta falcígera sesquigonfa; 23 , seta espinígera homogonfa; 24,25 setas espinígeras sesquigonfas. Escalas: $100 \mu \mathrm{m}$, figs. 19-21; $50 \mu \mathrm{m}$, figs. 22-25.

olhos conspícuos, dispostos em trapézio na metade posterior do prostômio; primeiro par em forma de meia-lua, segundo par circular; quatro pares de cirros tentaculares curtos, com o mais longo atingindo o setígero 2. Probóscide parcialmente evertida; o estado de conservação do espécime não permitiu a visualização das áreas em separado. Região dorsal lisa, sem elevações ou lamelas. Dois primeiros parapódios unirremes (fig. 19) e todos os demais birremes. Notopódios representados por cirro dorsal e lóbulo, sem lígulas. Cirro dorsal cilíndrico e longo, com o cirróforo expandido do setígero 10 ao 18 (fig. 20). Lóbulo notopodial cilíndrico aproximadamente do mesmo tamanho do cirro em parapódios anteriores, mais curto que este em parapódios medianos e posteriores (figs. 20, 21). Neuropódio com lóbulo pós-setal arredondado, achatado mais curto que o lóbulo notopodial 
e que a lígula neuropodial inferior (fig. 21). A partir dos setígeros 17-18 lóbulo neuropodial passa a acicular (fig. 22). Lígula neuropodial arredondada em parapódios anteriores e afilada em parapódios medianos e posteriores. Cirro ventral duplo a partir do setígero 1 , onde o ramo inferior é extremamente delicado e de difícil visualização. A partir do $3^{\circ}, 4^{\circ}$ setígero apresenta-se com $1 / 3$ do comprimento do ramo superior, aumenta gradativamente nos parapódios anteriores e medianos, até atingir aproximadamente o mesmo tamanho do superior em parapódios posteriores. Notosetas supra-aciculares homogonfas espinígeras e notosetas subaciculares homo- e sesquigonfas espinígeras (figs. 23-25). Neurosetas supra- e subaciculares homo- e sesquigonfas espinígeras e sesquigonfas falcígeras. As neurosetas falcígeras estão presentes do 1 ao $6^{\circ}-9^{\circ}$ setígeros. Os cinco primeiros parapódios apresentam neuroacículas extremamente robustas e escuras.

Diagnose. Quatro pares de cirros tentaculares, probóscide lisa e mandíbulas denteadas. Prostômio mais longo do que largo com um par de olhos. Lígula notopodial ausente. Cirro ventral duplo a partir do setígero1, com o ramo ventral apresentando $1 / 4$ do comprimento do ramo ventral. Cirróforos expandidos dos setígeros 10-18. Notosetas supra-aciculares homogonfas espinígeras e subaciculares homogonfas e sesquigonfas espinígeras. Região dorsal lisa. Neurosetas supra-aciculares espinígeras homogonfas; neurosetas subaciculares homo- e sesquigonfas espinígeras e falcígeras. As falcígeras aparecem nos primeiros setígeros e estão restritas à região anterior.

Comentários. A espécie semelhante, em termos de morfologia parapodial, tipo e distribuição de setas, é C. setosa Hutchings \& Reid, 1990, que apresenta cirro ventral bífido a partir do setígero 3, setas neuropodiais falcígeras e uma papila neuropodial. $C$. setosa foi descrita a partir de único exemplar, que se encontra em mau estado de conservação, o que impede exames morfológicos mais detalhados ou maiores discussões. A presença de cirro ventral duplo a partir do setígero 1, a presença de setas falcígeras nos 6-9 primeiros setígeros e a região dorsal lisa são tão distintivas em $C$. rocaensis, quando comparadas com as espécies congêneres, que justificam a eleição de uma nova espécie.

Ocorrência. Em poças de maré com substrato calcário.

Material-tipo. BRASIL, holótipo, Rio Grande do Norte: Atol das Rocas, (MCEM 1006); parátipos (MCEM 1006a, 5 exs).

\section{Laeonereis acuta (Treadwell, 1923)}

(Figs. 26-32)

Nereis (Leptonereis) acuta TREADWELL, 1923:1237, figs. 1-5.

Laeonereis culveri; HARTMAN, 1945: 21; PetTIBONE, 1971:14-19, figs. 5-7 [em parte].

Laeonereis pandoensis; ORENSANZ \& EsTIVARIZ, 1971:99, figs. 10-22.

Laeonereis acuta; ORENSANZ \& GIANUCA, 1974:9.

Comentários. Nereis (Leptonereis) acuta, coletada em Santos e descrita por Treadwell (1923), foi transferida para o gênero Laeonereis por Hartman (1945) e considerada um sinônimo júnior de Laeonereis culveri (Webster, 1880) por HARTMAN (1945) e Pettibone (1971). Esta última autora revisou o gênero e descreveu diferenças acentuadas na proporção entre as lígulas e os lóbulos notopodiais posteriores em espécimes provenientes da Flórida e da Geórgia. Os primeiros apresentam lígulas e lóbulos 

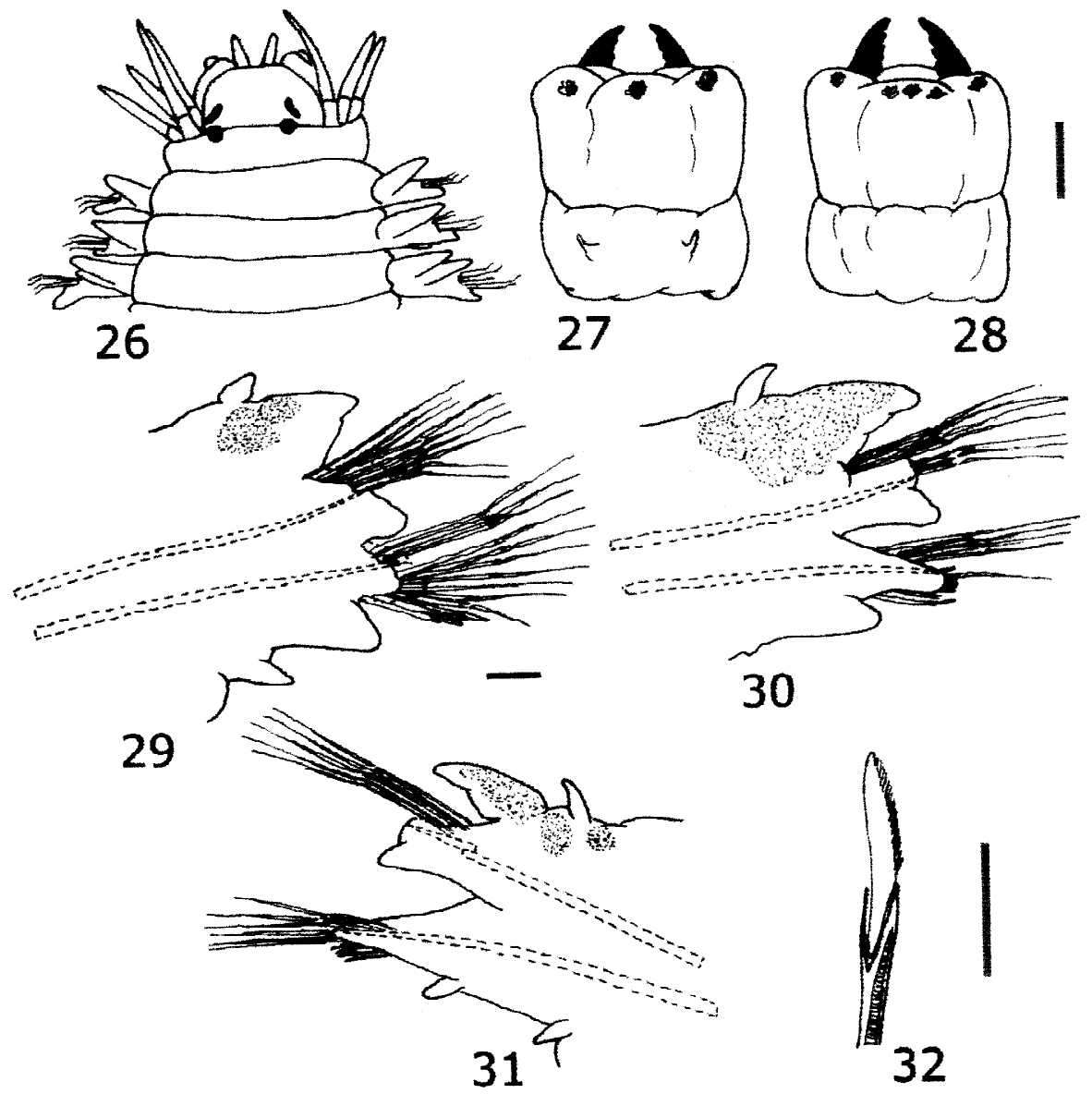

Figs. 26-32. Laeonereis acuta (Treadwell, 1923), rio Piauí, Sergipe: 26, região anterior, vista dorsal; 27, probóscide, vista dorsal; 28, probóscide, vista ventral; 29, parapódio do setígero 5, vista posterior; 30, parapódio do setígero 17, vista posterior; 31, parapódio do setígero 30, vista anterior; 32, seta falcígera homogonfa. Escalas: $100 \mu \mathrm{m}$, figs. 26-28; $500 \mu \mathrm{m}$, figs. 29-31; $50 \mu \mathrm{m}$, fig. 32 .

notopodiais aproximadamente do mesmo tamanho, com as lígulas maiores que os lóbulos, ambos triangulares. Os espécimes provenientes da Geórgia apresentam a lígula notopodial duas a três vezes mais longa que o lóbulo, ambos com formato mais delgado e acuminado. Apesar das variações, o gênero foi considerado monoespecífico, constituído por Laeonereis culveri, cuja distribuição iria da Flórida até o Uruguai. Orensanz \& Gianuca (1974) registraram diferenças na proporção entre os lóbulos notopodiais posteriores entre os espécimes subtropicais da América do Norte e da América do Sul. Nos exemplares sulamericanos identificados, os lóbulos notopodiais posteriores são aproximadamente do mesmo tamanho e nos espécimes norte-americanos o lóbulo notopodial superior é sempre 
mais longo. Consideraram também que existem diferenças quanto à biologia reprodutiva e a partir dessas constatações removeram $N$. (L). acuta da sinonímia de $L$. culveri reconhecendo duas espécies para o gênero: L. culveri, com distribuição de New Jersey ao Golfo do México e L. acuta de Santos a Península Valdés, na Argentina. L. pandoensis (Monro, 1937) foi considerada sinônimo júnior de L. acuta por Orensanz \& GianuCA (1974).

Em alguns indivíduos adultos coletados na Bahia, a região anterior do corpo é uniformemente cilíndrica, diferente do que ocorre em L. acuta, que tem o peristômio marcadamente estreito em relação à região anterior do corpo (fig. 26). Os exemplares provenientes do nordeste do Brasil apresentam a distribuição das papilas (figs. 27, 28), lígulas notopodiais posteriores aproximadamente do mesmo tamanho dos lóbulos, ligeiramente mais curtas nos últimos setígeros (figs. 29-31), e setas falcígeras homogonfas (fig. 32) com padrão semelhante ao dos exemplares das costas sudeste e sul do Brasil, referidos a $L$. acuta, o que amplia a distribuição da espécie para o norte.

Ocorrência. Em substrato arenoso na região oligohalina de estuários.

Distribuição. Atlântico Ocidental, de Sergipe, Brasil, ao Uruguai.

Material examinado. BRASIL, Sergipe: Estuário do rio Real (MCEM 1155-1208, 1047 exs); Bahia: baía de Todos os Santos (MCEM 1209, 6 exs).

\section{Rullierinereis auxiliadorae sp. nov.}

(Figs. 33-40)

Etimologia. A espécie foi nomeada em homenagem à Dra. Maria Auxiliadora Santos, que por muitos anos emprestou o seu entusiasmo, na Universidade Federal de Sergipe, àqueles dispostos a entrarem no mundo dos poliquetas.

Descrição. O holótipo é um espécime completo com 50 setígeros e $10 \mathrm{~mm}$ de comprimento total, medindo $450 \mu \mathrm{m}$ de largura sem os parapódios e $750 \mu \mathrm{m}$ com os parapódios. Coloração amarelo-claro em álcool 70\%. O setígero 2 apresenta uma banda dorsal marrom, larga no centro e estreita lateralmente, em direção aos parapódios. Prostômio triangular, duas vezes mais longo do que largo; um par de antenas frontais, cirriformes, ligeiramente mais longas do que os palpos; dois pares de olhos marrons, redondos, do mesmo tamanho e dispostos em retângulo na metade posterior do prostômio; linha vertical de pigmentação entre os olhos e palpos com palpóforos e palpóstilos cilíndricos; peristômio duas vezes mais longo que o setígero 1; quatro pares de cirros tentaculares, finos e curtos, o mais longo atinge o setígero 2 e o mais curto não chega ao setígero 1 (fig. 33). Probóscide lisa. Cirro dorsal inserido na base do lóbulo notopodial triangular, aproximadamente do mesmo tamanho deste em parapódios anteriores e mais longo que este em parapódios medianos e posteriores; lígula notopodial ausente ao longo de todo o corpo (figs. 34, 36). Lóbulo notopodial triangular e aproximadamente do mesmo tamanho do lóbulo neuropodial em parapódios anteriores, mais curto em parapódios medianos e posteriores (figs. 35, 36). Lóbulo neuropodial triangular aproximadamente do mesmo tamanho da lígula neuropodial cônica (figs. 35, 37). Cirro ventral cônico, aproximadamente do mesmo tamanho da lígula neuropodial nos setígeros anteriores (fig. 34) e mais curto que esta em setígeros medianos e posteriores. Notosetas espinígeras homogonfas (fig. 37) até o setígero 15; a partir do setígero 16 são substituídas por uma seta falcígera 


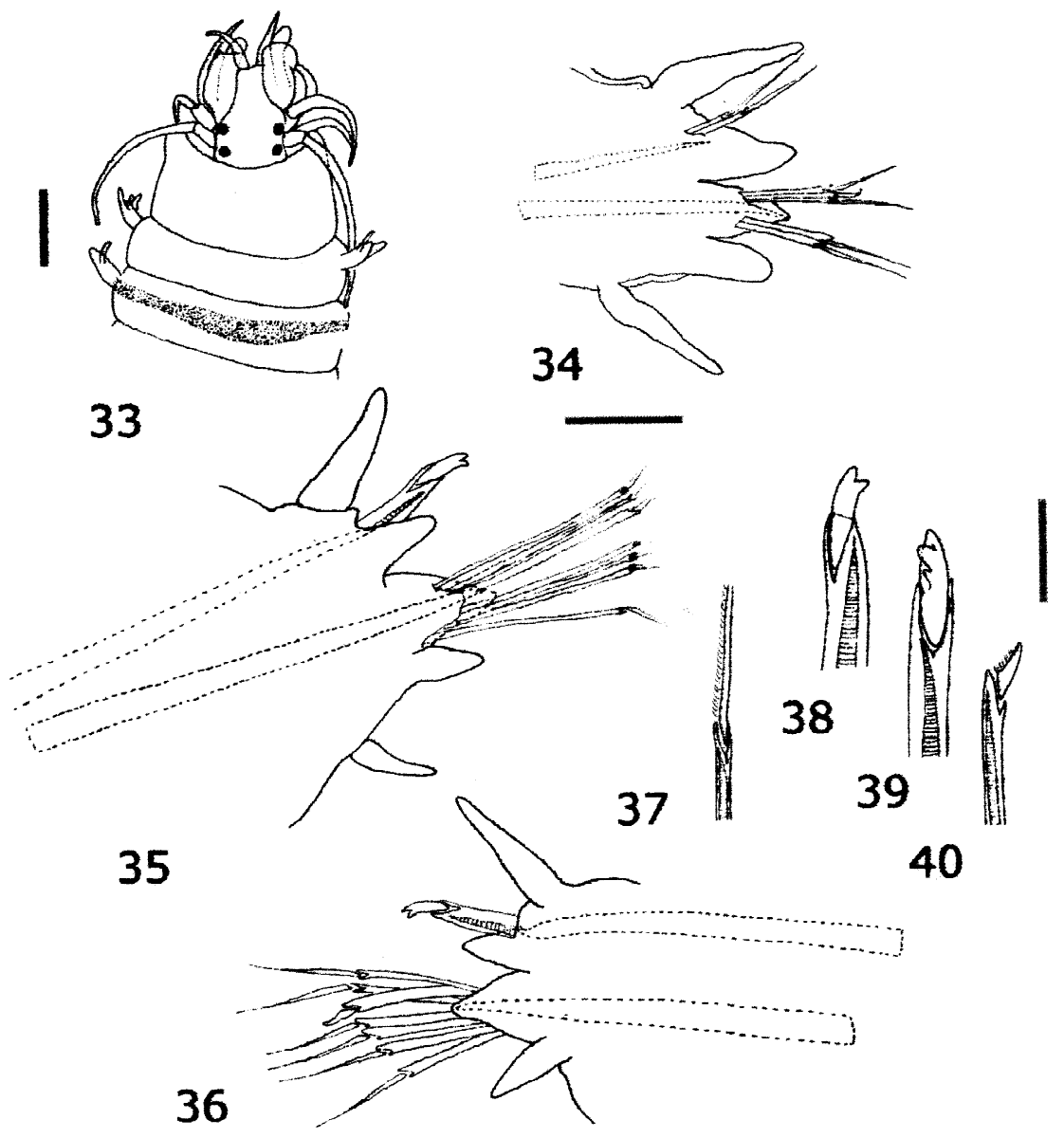

Figs. 33-40. Rullierinereis auxiliadorae sp. nov., holótipo: 33, região anterior, vista dorsal; 34, parapódio do setígero 7, vista posterior; 35, parapódio do setígero 17, vista anterior; 36, parapódio do setígero 40, vista anterior; 37, seta homogonfa espinígera; 38 , seta falcígera homogonfa bidentada; 39 , seta falcígera homogonfa tridentada; 40, seta falcígera heterogonfa. Escalas: $100 \mu \mathrm{m}$, figs. 33-36; $50 \mu \mathrm{m}$, figs. 37-40.

homogonfa robusta, bi- ou tridentada (figs. 38, 39). Neurosetas supra-aciculares espinígeras homogonfas e falcígeras heterogonfas; neurosetas subaciculares espinígeras e falcígeras heterogonfas (fig. 40). Pigídio com um par de uritos finos, que atingem o comprimento dos dois últimos setígeros.

Diagnose. Quatro pares de cirros tentaculares, probóscide lisa e mandíbulas denteadas. Margem anterior do peristômio cobrindo o primeiro par de olhos, que permanece visível por transparência. Lígula notopodial ausente. Região dorsal com faixa de pigmentação transversal no segundo setígero (fig. 33). Cirro dorsal cilíndrico, aproximadamente do mesmo tamanho ao longo do corpo, cirro ventral cônico similar à 
lígula neuropodial em comprimento nos primeiros setígeros e diminuindo gradativamente (figs. 34, 36). Notosetas homogonfas espinígeras e homogonfas falcígeras, bi- e tridentadas (figs. 38, 39). Neurosetas supra-aciculares espinígeras homogonfas e falcígeras heterogonfas; neurosetas subaciculares espinígeras e falcígeras heterogonfas. Comentários. Rullierinereis misakiensis (Imajima \& Hayashi, 1969) difere de $R$. auxiliadorae, por apresentar seta notopodial falcígera homogonfa exclusivamente tridentada, cirro dorsal duas vezes mais longo que a lígula notopodial em parapódios posteriores, lóbulo notopodial posterior maior do que o lóbulo neuropodial e cirros tentaculares longos alcançando o setígero 4. R. bahamensis (Hartmann-Schröder, 1958) apresenta papilas proboscidiais e setas tridentadas em parapódios anteriores. LiÑEROARAÑA \& VÁsQuEZ (1979) descreveram os parapódios posteriores de R. bahamensis com quatro projeções, considerando três delas bem-desenvolvidas e uma quarta, a notopodial superior, como incipiente. Entretanto, as figuras (L.-ARAÑa \& VÁSQUEZ, 1979:5, figs. 1.5 e 1.6) mostram a mesma projeção um pouco mais curta que o lóbulo notopodial, o que permitiria descrevê-la como uma lígula notopodial com cirro dorsal inserido na região terminal.

Ocorrência. Em fundos de plataforma, entre 18 e $21 \mathrm{~m}$, em substrato calcário.

Material-tipo. BRASIL, holótipo, Ceará: Projeto GEOCOSTA I (MCEM 1213); parátipos: Projeto GEOCOSTA (MCEM 1211-1214, 6 exs).

Agradecimentos. A todos aqueles que gentilmente cederam nereidídeos para este estudo: Verônica Oliveira (Universidade Federal do Maranhão), Wilson Franklin (Universidade Federal do Ceará), Rosângela Gondim (Universidade Federal do Rio Grande do Norte), Martin Christoffersen e Elineí Araújo (Universidade Federal da Paraíba), Bety Rose (Universidade Federal Rural de Pernambuco), Tereza Callado (Universidade Federal de Alagoas), Maria Auxiliadora Santos, Erminda Couto, Wellington Sousa e Denise Santos (Universidade Federal de Sergipe), Lêda Santa-Isabel (Universidade Federal da Bahia), Paulo César de Paiva (Universidade Federal Rural do Rio de Janeiro) e Sérgio Netto (UNISUL, Santa Catarina).

\section{REFERÊNCIAS BIBLIOGRÁFICAS}

BANSE, K. 1977. Gymnonereidinae new subfamily: the Nereididae (Polychaeta) with bifid parapodial neurocirri. J. Nat. Hist., London, 11:609-628.

CorrêA, D. D. 1948. A polychaete from the Amazon region. Bolm Fac. Filos. Ciênc. Let. Univ. S. Paulo, Sér. Zool., São Paulo, 13:245-252.

DAY, J. H. 1967. A monograph on the Polychaeta of Southern Africa: Part I Errantia. London, Bristish Museum (Natural History). 374p.

1973. New Polychaeta from Beaufort, with a key to all species recorded from North Carolina. NOAA Technical Reports, Washington, 375:1-140.

Glasby, C. J. 1999. The Namanereidinae (Polychaeta: Nereididae). Part 1. Taxonomy and phylogeny. Rec. Aust. Mus., Sydney, 25(Suppl.):1-129.

GruBE, A. E. 1872. Ueber die Gattung Lycastis und paar neue Arten derselben. Jber. schles. Ges. vaterl Kult., Breslau, 49:47,48.

HANSEn, A. 1882. Recherches sur les Annélides recueillies par M. le ProfesseurÉdouard Van Beneden pendant son voyage au Brésil et à la Plata. Mèm. Acad. R. Sc. Belg., Brussels, 44:1-29.

Hartman, O. 1945. The marine annelids of North Carolina. Duke Univ. Mar. St. Bull., Durham, 2:1-51. 1959. Capitellidae and Nereidae (Marine Annelids) from the Gulf Side of Florida, with reviews of freshwater Nereidae. Bull. mar. Sci. Gulf . Caribb., Coral Gables, 9 (1-4):153-168.

Holme, N. A. \& McIntyre, A. D. 1971. Methods for the study of marine benthos. 1ed. Oxford, Blackwell Scientific. 334p.

Hutchings, P. \& Turvey, S. P. 1982. The Nereididae of South Australia. Trans. R. Soc. S. Aust., Adelaide, 106:93-144. 
Kinberg, J. 1866. Annulata nova. Öfver K. VetenskAkad. Förh., Stockholm, 22:167-179.

LANA, P. C. 1987. Padrões de distribuição geográfica dos poliquetas errantes (Annelida: Polychaeta) do Estado do Paraná. Ciênc. Cult., São Paulo, 39:1060-1063.

Lana, P. C. \& Sovierzoski, H. H. 1987. Neanthes bruaca sp. n. e Nereis broa sp. n. (Nereididae Polychaeta) da costa sudeste do Brasil. Arq. Biol. Tecnol., Curitiba, 30(4):677-688.

Liñero-Arana, I. \& VÁsquez, G. R. 1979. Nereidae (Polychaeta, Errantia) del Golfo de Cariaco, Venezuela. Boln Inst. Oceanogr. Univ. Oriente, Cumana, 18 ( 1/2):3-12.

Malmgren, A. J. 1867. Annulata Polychaeta Spetsbergiae, Groenlandiae, Islandiae et Scandinaviae hactenus cognita. Öfver K. VetenskAkad. Förh., Stockholm, 24:127-235.

Morgado, E. H. \& AmARAL, A. C. Z. et al. 1994. Intertidal sandy beaches polychaetes of São Sebastião Island, southern Brazil. Mém. Mus. natn. Hist. nat. Paris, Paris, 162:485-492.

Nonato, E. \& LunA, J. A. C. 1970. Anelídeos poliquetas do Nordeste do Brasil: I. Poliquetas bentônicos da costa de Alagoas e Sergipe. Bolm Inst. Oceanogr. São Paulo, São Paulo, 19 (1):57-130.

Orensanz, J. M. \& Estivariz, M. C. 1971. Los anelideos poliquetos de aguas salobres de la Província de Buenos Aires. Revta Mus. La Plata, Zool., La Plata, 11 (98):95-114.

OrensanZ, J. M \& GianUCA, N. M. 1974. Contribuição ao conhecimento dos anelídeos poliquetas do Rio Grande do Sul, Brasil. I. Lista sistemática preliminar e descrição de três novas espécies. Comun. Mus. Ciênc. PUCRS, Porto Alegre, 4:1-37.

Paiva, P. C. 1993. Anelídeos poliquetas da plataforma continental norte do Estado de São Paulo. I. Padrões de densidade e diversidade específica. Bolm Inst. Oceanogr. São Paulo, São Paulo, 41 (1/2):69-80.

Pettibone, M. H. 1971. Revision of species referred to Leptonereis, Nicon, and Laeonereis (Polychaeta: Nereididae). Smithson. Contr. Zool., Washington, 104:1- 53.

ReBelo, F. C. 1988. Primeira ocorrência do poliqueta estuarino Namalycastis abiuma (Muller, in Grube, 1871) na baía de Guanabara, Rio de Janeiro (Polychaeta, Nereidae). Incluindo notas de laboratório. Bolm Univ. Fed. Juiz de Fora, Juiz de Fora, 41:3-12.

Rullier, F. \& Amoureux, L. 1979. Annélides polychètes. Résultats scientifiques des campagnes de la Calypso. Ann. Inst. Océanogr., Monaco, 55(Suppl.):10-206.

Santos, C. S. G. \& Lana, P. C. 2000. Nereididae (Annelida; Polychaeta) da costa nordeste do Brasil. I. Padrões regionais e zoogeográficos de distribuição. Iheringia, Sér. Zool., Porto Alegre, (88):181-189.

Santos, M. A.; Santos, C. S. G. \& Oliveira, C. M. M. 1994. Polychaeta in the estuary of the Piauí river, Sergipe, Brazil. Mém. Mus. natn. Hist. nat., Paris, 162:541-547.

TAYLOR, J. L. 1984. Family Nereidae Johnston, 1845. In: Uebelacker, J. M. \& Johnson, P. G. Taxonomic guide to the polychaetes of the northern Gulf of México. Mobile, Barry A. Vittor \& Associates. v.5 p.1- 42.

Treadwell, A. L. 1920. A new polychaetous annelid of the genus Nereis from Brazil. Proc. U. S. natn. Mus., Washington, $\mathbf{5 8}$ (2347):467-468.

. 1923. Two new species of polychaetous annelids of the genus Nereis from Brazil. Revta Mus. Paulista, São Paulo, 13:1237-1243.

1932. New species of polychaetous annelids from Ilha de São Sebastião, together with a list of other annelids determined from Brasil. Revta Mus. Paulista, São Paulo, 17 (2):13-22.

Wu, B.; Ruiping, S. Y. \& YAng, D. J. 1985. The Nereidae (Polychaetous Annelids) of the Chinese coast. Beijing Ocean. $234 \mathrm{p}$.

Recebido em 05.09.2000; aceito em 13.07.2001 